\title{
Evaluation of limited blood sampling population input approaches for kinetic quantification of $\left[{ }^{18} \mathrm{~F}\right] \mathrm{fluorothymidine}$ PET data
}

Kaiyumars B Contractor ${ }^{1}$, Laura M Kenny ${ }^{1}$, Charles R Coombes ${ }^{1}$, Federico E Turkheimer ${ }^{2}$, Eric O Aboagye ${ }^{1,3^{*}}$ and Lula Rosso ${ }^{2}$

\begin{abstract}
Background: Quantification of kinetic parameters of positron emission tomography (PET) imaging agents normally requires collecting arterial blood samples which is inconvenient for patients and difficult to implement in routine clinical practice. The aim of this study was to investigate whether a population-based input function (POP-IF) reliant on only a few individual discrete samples allows accurate estimates of tumour proliferation using $\left[{ }^{18} \mathrm{~F}\right]$ fluorothymidine (FLT).

Methods: Thirty-six historical FLT-PET data with concurrent arterial sampling were available for this study. A population average of baseline scans blood data was constructed using leave-one-out cross-validation for each scan and used in conjunction with individual blood samples. Three limited sampling protocols were investigated including, respectively, only seven (POP-IF7), five (POP-IF5) and three (POP-IF3) discrete samples of the historical dataset. Additionally, using the three-point protocol, we derived a POP-IF3M, the only input function which was not corrected for the fraction of radiolabelled metabolites present in blood. The kinetic parameter for net FLT retention at steady state, $\mathrm{K}_{\mathrm{i}}$, was derived using the modified Patlak plot and compared with the original full arterial set for validation.
\end{abstract}

Results: Small percentage differences in the area under the curve between all the POP-IFs and full arterial sampling IF was found over 60 min (4.2\%-5.7\%), while there were, as expected, larger differences in the peak position and peak height.

A high correlation between $\mathrm{K}_{\mathrm{i}}$ values calculated using the original arterial input function and all the populationderived IFs was observed $\left(R^{2}=0.85-0.98\right)$. The population-based input showed good intra-subject reproducibility of $K_{i}$ values $\left(R^{2}=0.81-0.94\right)$ and good correlation $\left(R^{2}=0.60-0.85\right)$ with Ki-67.

Conclusions: Input functions generated using these simplified protocols over scan duration of 60 min estimate net PET-FLT retention with reasonable accuracy.

Keywords: FLT, Kinetic modelling, Input function, Quantification, Tumour proliferation

\section{Background}

Positron emission tomography (PET) imaging with $\left[{ }^{18} \mathrm{~F}\right]$ fluorothymidine (FLT-PET) is believed to provide an in vivo measurement of tumour proliferation [1]. FLT is a substrate for the cell-cycle dependent enzyme, thymidine kinase (TK1), and its accumulation in cells is

\footnotetext{
* Correspondence: eric.aboagye@imperial.ac.uk

'Department of Surgery and Cancer, Imperial College Healthcare NHS Trust, Hammersmith Hospital, London, UK

Full list of author information is available at the end of the article
}

proportional to the activity of this enzyme, which in turn is correlated with sustained cellular proliferation. The accumulation of FLT in cells, therefore, provides an indication of their proliferation rate [2], and a high positive correlation with Ki-67 labelling index (Ki-67), an immunohistochemical marker for cellular proliferation, has been found [3-6]. Although semi-quantitative measures, like the standard uptake value (SUV), can be obtained from both static and dynamic PET images, only a full kinetic analysis will provide direct 
information on the rate of FLT phosphorylation. Importantly, a quantitative measure of tumour FLT flux may be necessary when evaluating novel anti cancer therapies for which the effect on kinetics is unknown.

Key physiological parameters of PET tracer kinetics can be quantified using models which usually require an input function (IF)-a measure of the amount of radioactivity that enters the organ of interest. As FLT undergoes glucuronidation predominantly in the liver, the fraction of the FLT-glucuronide radiolabelled metabolite present in plasma needs also to be monitored and used to derive the metabolite corrected input function. Peripheral arterial blood sampling during the time of the scan has been extensively used for this purpose and has been shown to achieve the desirable precision in modelling the kinetics of radiotracers. In order to obtain an accurate profile of radioactive blood concentration in the first 10 min after tracer injection, continuous sampling is often used. However, arterial cannulation has a number of drawbacks which limit the number of blood samples that could be obtained and prohibit the use of continuous sampling in some groups, particularly in cancer patients who are often anaemic. This fact, combined with the complexity and high cost of PET measurements, makes it highly desirable to validate alternative approaches for obtaining input functions and maximise the information obtained.

When the PET images include regions of activity of a prominent blood vessel, like the aorta, these may be sampled to derive a blood pool IF that can be used as replacement for continuous sampling for the early period when radioactive spill over from surrounding tissue is limited. However, because of the small size of these regions, which is often inferior or comparable to the resolution of the PET scan, the image-derived input function methods require careful correction for partial volume effects [7-11] and still necessitate a number of blood samples for metabolites analysis if the tracer is metabolised during the scan.

Recently, there has been interest in the use of limited arterial blood sampling methods [12-14] that explore the possibility of using fewer samples, albeit with a loss of accuracy, but leading to better clinical compliance. Additionally, to avoid individual continuous sampling and account for blood sampling errors, a population-based input function may be determined. This involves pooling together previously collected arterial blood data and calculating the average blood response to radiotracer injection. Subject specific calibration of the mean blood curve can be performed using a few individual discrete blood samples. The population averaged input function has been described in neurological and some oncology studies using FDG and shows promise [15-18].
To date, relatively few limited sampling methodologies (four to eight samples for blood radioactivity concentration and at least one sample for metabolite analysis) have been proposed for FLT-PET [14,19-22], and FLT kinetics has been recently estimated using image-derived input function in colorectal cancer [21], non-small-cell lung cancer [22] and high grade gliomas [23]. In this study, we investigated the consequences of using a population input approach and highly reduced sampling protocols to estimate FLT kinetics in oncology PET studies without continuous arterial sampling. Previously acquired FLT-PET scans with concurrent continuous arterial data were used to validate the results. We then assessed whether test-retest variability and accuracy for determination of proliferation of the population input functions were affected by this reduced sampling population-based methodology.

\section{Methods}

\section{FLT data set}

The data set comprised of 15 breast cancer patients who underwent three dynamic FLT PET scans. Of the 15 cases, 9 cases underwent two pre-treatment scans within 2-8 days of each other before starting chemotherapy treatment. Baseline tumour proliferation indices Ki-67 were available in 12 of the 15 patients with suitable histology. In the present study, only the primary lesion was analysed for each patient. The reproducibility, as well as correlation with $\mathrm{Ki}-67$, has been previously published $[5,24]$. The study was approved by the local Hospital Ethics committee and the Administration of Radioactive Substances Advisory Committee, United Kingdom. All patients gave written informed consent to take part.

\section{Blood data acquisition and analysis of original data set}

Patients were scanned in the Siemens ECAT ${ }^{\circledR} 962 \mathrm{HR}^{+}$ PET scanner (CTI/Siemens, Knoxville, TN, USA)). Dynamic PET scanning was commenced after injection of an average of $370 \mathrm{MBq}$ of FLT as a bolus and lasted 90 min. Arterial blood sampling was performed continuously for the first 10 min of scanning, and then discrete arterial samples were taken at baseline and after 2.5, 5, 10, 20, 30, 45, 60, 75 and 90 min. Continuous blood sampling was counted in an in-house bismuth-germanateoxide counter to record the initial blood sampling radioactivity. For the discrete samples, the total blood radioactivity and plasma radioactivity were determined by gamma counter (Raytek, Sheffield, UK), and the plasma parent (FLT) fraction was determined by HPLC with radiochemical detection (Acrodisc, VWR International Ltd., Leicestershire, UK) [5,24] at 2.5, 5, 10, 30, 45, 60, 75 and 90 min after scan start. All data were fitted using non-linear least square minimisation using Matlab ${ }^{\circledR}$ based on the interior-reflective Newton method $[25,26]$. 
The analytical functions for the optimisation were chosen as the one that produced the best agreement, for each given reduced sampling protocol, with the original blood fits. All blood and plasma data belonging to the same type of POP-IFs were fitted using the same functions to ensure consistency within the protocol. The parent fraction was modelled using a sigmoid function, the plasma over blood ratio using an exponential approach to a constant (only the first $10 \mathrm{~min}$ ), and the whole series of plasma and blood data points using a cubic spline interpolation, see Appendix. Although FLT kinetic constants can be determined with more accuracy using $90 \mathrm{~min}$ of dynamic acquisition [27], $60 \mathrm{~min}$ acquisition have been used to quantify accurately FLT retention at steady state $\left(\mathrm{K}_{\mathrm{i}}\right)$ [14] and would be more advantageous in routine clinical settings. Only 0-60 min arterial blood data of the available $90 \mathrm{~min}$ was used for this validation study (i.e. continuous sampling plus seven discrete samples up to $60 \mathrm{~min}$, henceforth, referred to as the "full arterial sampling" set or FA).

\section{Generation of the population input function}

Only the total blood IFs of pre-treatment scans were used to derive the initial part of the POP-IFs (up to 2.5 min) for both pre-treatment and post-treatment scans. Each blood input function was divided by the individual scan injected dose, and to avoid information overlap, we excluded for each patient his/her relative original blood input function(s) and constructed an individualised average normalised total blood input function (POP-IF) for each patient, see Figure 1. Then for each scan, a new population-based parent plasma IF was generated by multiplying the individual scan injected dose of FLT by

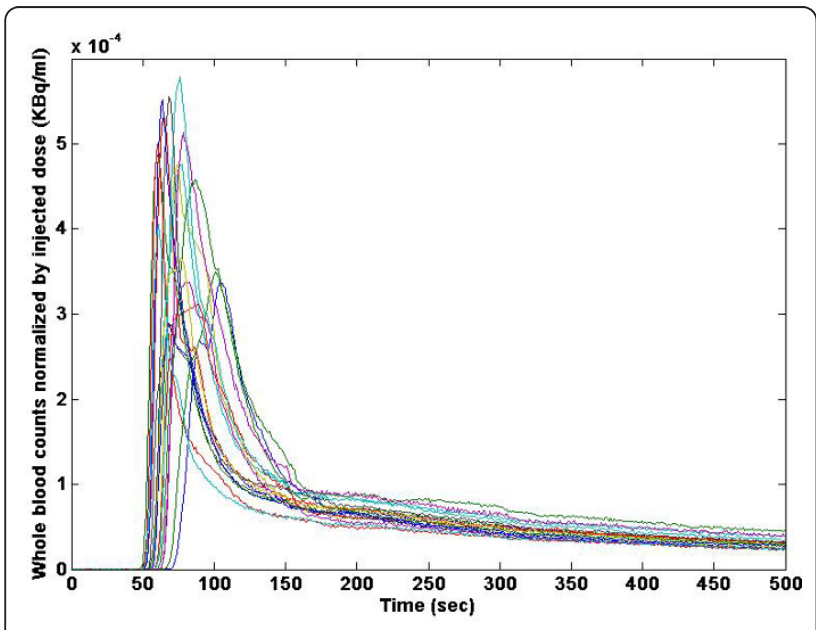

Figure 1 Whole blood time curves from 36 FLT scans normalised by injected dose. Arterial blood sampling was performed continuously for the first 10 min of scanning. Note the population variance. the dose-normalised average of the leave-one-out pretreatment subset and corrected by the plasma over blood ratio up to $2.5 \mathrm{~min}$, see Appendix. Correction by subject weight has also been considered, but did not influence the results in this dataset.

The latter part of the POP-IF, after the first sampling data point, was derived by interpolating the individual plasma radioactivity concentration measurements and scaling it by the unchanged FLT fraction modelled using one of three reduced blood sampling protocols as described in the next paragraph. Only in the case of the POP-IF3M the metabolite correction was not performed.

\section{Reduced blood sampling protocols}

To test whether adequate kinetic estimation could be obtained using IF corrected by fewer blood samples, three reduced sets of discrete blood sample data were formulated. The original blood data was abbreviated and several combinations were tested to determine the optimal time points that would best reproduce the original arterial input in a reduced sampling scenario. A full optimization of the sampling times was outside of the scope of this study based on historical data. The first set consisted of all seven discrete samples (up to $60 \mathrm{~min}$ ) from the original full arterial sampling set, namely at baseline, 2.5, 5, 10, 20, 30, 45 and 60 min post injection of FLT. The population-based input function we derived using this protocol was called POP-IF7. The second set consisted of only five discrete arterial blood samples taken from the original set at 2.5, 5, 10, 30 and $60 \mathrm{~min}$ after the start (POP-IF5), and the third set comprised of three samples taken from the original set at times 2.5, 10 and 45 min after start of FLT (POP-IF3). The plasma over blood ratio was modelled using a least square fit to an exponential function leading to a constant to correct the average blood curve. The plasma radioactivity concentration as a function of time were interpolated using a least square fit to a two exponential function plus a constant when three points were available, and a cubic spline plus a constant when five points were available. The resulting plasma input function was corrected for the presence of metabolites. The parent fraction curve was modelled using an exponential approach to a constant when only three points were used, and a sigmoid when five points were available, see Appendix.

Finally, we evaluated the possibility of further simplifying the blood protocol, generating a population-based input function following the same procedure of the POP-IF3 but uncorrected for the contribution of metabolites. This input function was called POP-IF3M. A summary of all population-based input functions is presented in Table 1. Quantification of the differences in the population-derived input functions with respect to 
Table 1 Summary of blood measurements used to generate the population input functions evaluated in this study

\begin{tabular}{|c|c|c|c|c|c|}
\hline & $\begin{array}{l}\text { FA- } \\
\text { IF }\end{array}$ & $\begin{array}{l}\text { POP- } \\
\text { IF7 }\end{array}$ & $\begin{array}{l}\text { POP- } \\
\text { IF5 }\end{array}$ & $\begin{array}{l}\text { POP- } \\
\text { IF3 }\end{array}$ & $\begin{array}{l}\text { POP- } \\
\text { IF3M }\end{array}$ \\
\hline Continuous sampling & yes & no & no & no & no \\
\hline Number of blood samples & 7 & 7 & 5 & 3 & 3 \\
\hline $\begin{array}{c}\text { Number of metabolite } \\
\text { measures }\end{array}$ & 7 & 7 & 5 & 3 & 0 \\
\hline
\end{tabular}

the original full arterial sample procedure (i.e. continuous sampling for the first 10 min concurrent with seven discrete samples up to $60 \mathrm{~min}$ ) was determined by calculating the area under the curve (AUC), height of the peak and position of the peak (with respect to time) for all population-based input functions.

\section{Data analysis}

We wanted to assess how the POP-IFs affected the quantification of tumour FLT-PET uptake compared to using the original FA-IFs derived from the full arterial sampling standard approach. The kinetics of FLT were determined by calculating the net irreversible plasma to tumour transfer constant at steady state, $K_{i}$, the relevant kinetic parameter reflecting cellular proliferation [4]. This was done for all the four population-based input functions using the established modified Patlak's graphical method $[5,28]$ The percentage difference $( \pm$ standard deviation) between the population-based input functions and full arterial results was calculated, together with the within-subject variability and correlation with Ki-67.

\section{Results and discussion}

Population input function

All combined data from 36 FLT dynamic PET scans were available and analysed. The population average derived IFs, as expected, did not produce the same initial peak height (from 0-2 min) as that of the original obtained with full arterial sampling (Figure 2). However, after approximately $2 \mathrm{~min}$, the POP-IF curves were almost undistinguishable to that of the full arterial sampling method.

Due to these observations, the area under the plasmatic curve (AUC) was divided in two parts: AUC-1 was calculated between 0.0 and $2.5 \mathrm{~min}$, and AUC-2 was calculated from the first individual discrete sample (taken at $2.5 \mathrm{~min}$ for all protocols) to the end of the scan. The mean absolute percentage difference $( \pm$ standard deviation) between the full arterial sampling IF and the POP-IF was $30 \%( \pm 16 \%)$ for the peak height (highest point of the IF), $16 \%( \pm 20 \%)$ for the peak position (position with time on the $\times$ axis) and $13 \%( \pm 18 \%)$ for the area under the peak (AUC-1). However, the AUC-2 mean absolute percentage difference between the full
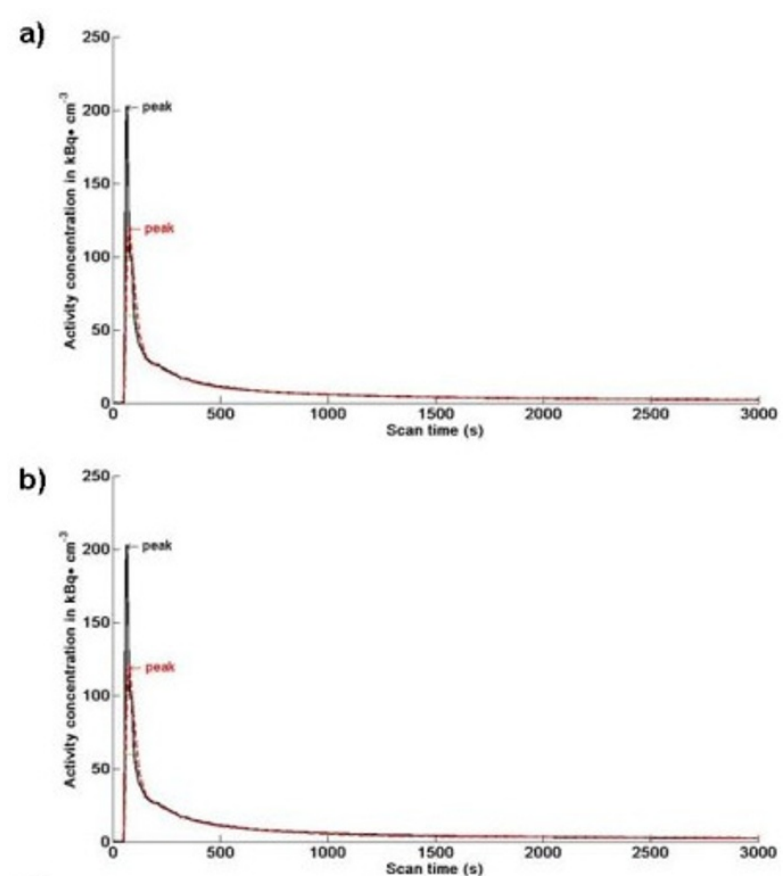

c)

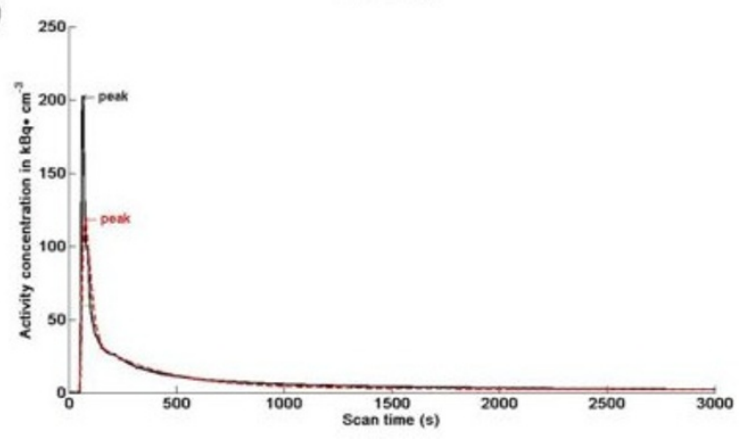

Figure 2 The comparison of subject 1 input function obtained using full arterial sampling and average POP-IFs. Comparison of subject 1 input function obtained using full arterial sampling (black curve) and average POP-IF (red curve) obtained using a) seven discrete samples up to 65 min (POP-IF7), b) five discrete samples (POP-IF5) and c) three discrete samples (POP-IF3). As expected, the population-based input functions failed to reproduce the true initial peak height. However, the curves superimposed well after 2 min post injection, where the POP-IF are based on the individual blood measures.

arterial sampling IF and the POP-IF was $1.5 \%( \pm 0.6 \%)$ using POP-IF7, 3.5\% $( \pm 2.5 \%)$ using POP-IF5 and $5.3 \%$ $( \pm 2.6 \%)$ using POP-IF3, indicating that the POP-IFs provide a reasonable approximation of the full arterial sampling IF in the latter part of the curve.

\section{Quantification of FLT uptake using the population based input functions}

We investigated the accuracy of FLT uptake quantification obtained with the three limited blood sampling protocols described. Using the modified Patlak modelling method 


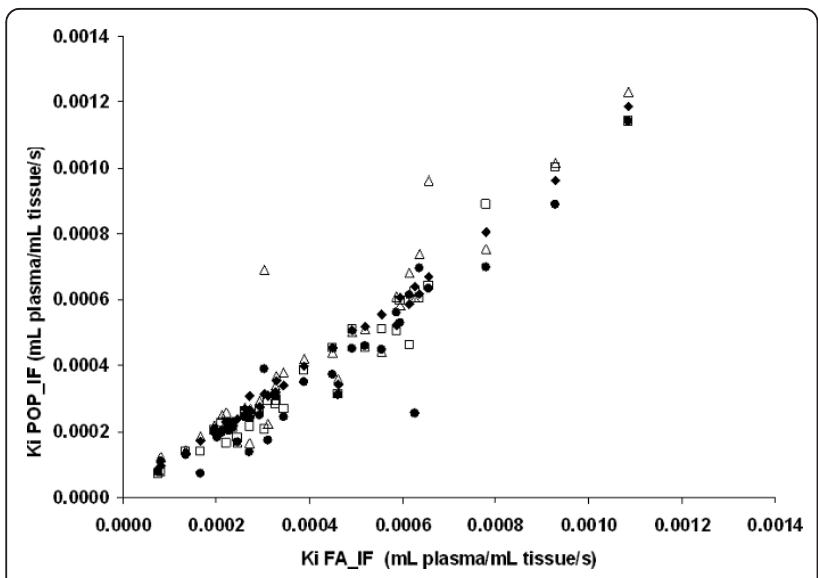

Figure 3 The correlation between FA-IF and POP-IFs modified Patlak $\mathbf{K}_{\mathbf{i}}$. Correlation between modified Patlak $K_{\mathbf{i}}$ using FA-IF and the Ki obtained using POP-IF7 (circles, $r^{2}=0.98$ ), POP-IF5 (squares, $r^{2}=$ 0.96), POP-IF3 (crosses, $r^{2}=0.88$ ) and POP-IF3M (triangles, $r^{2}=0.85$ ).

$[5,28]$, the mean absolute percentage difference between $\mathrm{Ki}$ values obtained using FA-IF and Ki obtained using POP-IF7 was $5.0 \%( \pm 5.2 \%)$, was $10.3 \%( \pm 9.1 \%)$ using POP-IF5, 12.8\% ( $\pm 13.1 \%)$ using POP-IF3, and 14.8\% ( \pm 14.3\%) using POP-IF3M. High correlation (Figure 3) was found between Ki values obtained by the full arterial sampling, and Ki obtained using the population-based input with seven discrete samples (POP-IF7, $\mathrm{R}^{2}=0.98$ ), five discrete samples (POP-IF5, $R^{2}=0.96$ ), three discrete samples (POP-IF3, $\mathrm{R}^{2}=0.88$ ) and three discrete sample without metabolite correction (POP-IF3M, $\mathrm{R}^{2}=0.85$ ). In patients with breast cancer, the standard deviation of $\mathrm{K}_{\mathrm{i}}$ estimated from FLT reproducibility studies (test-retest without treatment) using Patlak graphical analysis and FA-IF was 15\% [24]; hence, the estimation error obtained using the new POP-IFs with limited sampling was within the intrinsic estimation error of Ki.

FLT is used as a marker of proliferation, and retention of FLT is believed to be related to the phosphorylation of the radiotracer by TK1. Good correlation was found between POP-IF Ki values and a standard marker of proliferation, Ki-67: using seven discrete samples (POPIF7, $R^{2}=0.81$ ), using five discrete samples (POP-IF5, $R^{2}$ $=0.80$ ), using three discrete samples (POP-IF3, $\mathrm{R}^{2}=$ 0.64 ) and using three discrete samples without metabolite correction (POP-IF3M, $\mathrm{R}^{2}=0.60$, not shown), versus $R^{2}=0.85$ for FA-IF as shown in Figure 4. These values are superior or comparable to the correlation found between Ki-67 and SUV values in the same data set $\left(R^{2}=0.62\right)[5]$. For the patients who completed two pre-treatment scans, regression analysis showed a good test-retest correlation in all POP-IF protocols, namely $\mathrm{R}^{2}=0.92$ for POP-IF7, $\mathrm{R}^{2}=0.89$ for POP-IF5, $\mathrm{R}^{2}=0.90$ for POP-IF3, $R^{2}=0.81$ for POP-IF3M, versus $R^{2}=0.94$ for FA-IF.
FLT-PET net flux quantification may be needed to determine the effect of new drugs on tumour proliferation as semi quantitative measures like SUVs may be affected by changes in the blood flow. Kinetic quantification normally requires continuous concurrent monitoring of radioactivity in the blood. These measurements are invasive, costly and difficult to implement in routine clinical practice. In this investigation, we calculated the physiologically relevant rate constant $\mathrm{K}_{\mathrm{i}}$ in tumours by employing a limited discrete sampling approach without continuous arterial sampling. This has been possible by constructing a population input function (POP-IF) based on a leave-one-out cross-validated average of the pre-treatment scans subset for the initial part of the curve and on the individual plasma and metabolites data for the latter part. Although this population-based method could lead to inaccurate estimates of the rate constants describing initial kinetic events such as delivery of FLT, because of the larger individual variability of the initial peak of the plasma curves, we noted that the POP-IF curves (corrected either by seven, five or three individual discrete samples) were comparable to the full arterial IF curve approximately $2 \mathrm{~min}$ after injection of FLT, since in this part of the curve, they are based on the modelling of individual blood and plasma measurements. For this reason, the reduced sampling protocol did not affect the estimation of net transfer constant $K_{i}$ that depends on the $K_{1} / k_{2}$ ratio (with errors in $K_{1}$ likely reflected in $k_{2}$ and normalised in their ratio) and on the phosphorylation rate constant $k_{3}$ that is reflected on the late points in the curve. Note that $K_{i}$ was estimated using the established modified Patlak's graphical method. This simplified procedure does not derive the two compartment model's rate constants individually. We expect that a complete solution of the non-linear model would be more dependent on IF accuracy. The POP-IFs generated results in good agreement with the original FA-IFs for both pre-treatment and post-treatment scans. Treatment per se did not affect the IF. There was a high correlation between the values of $K_{i}$ derived using FA-IF and all the POP-IF protocols evaluated in this study $\left(R^{2}=0.85-0.98\right)$. The POP-IF Ki were also correlated with the percentage of Ki-67 positive cells $\left(R^{2}=0.81-0.60\right)$ and performed fairly well in reproducibility test $\left(R^{2}=0.81-0.92\right)$. The estimation errors increased with decreased number of samples because of the less accurate modelling of the individual blood and plasma curves.

This approach could be used for other ligands that are metabolised. To this end, the degree of metabolism will affect the robustness of the approach, with estimates of IF for minimally metabolised tracers being the most robust, particularly when POP-IF3M is used as an estimate. 


\section{a)}

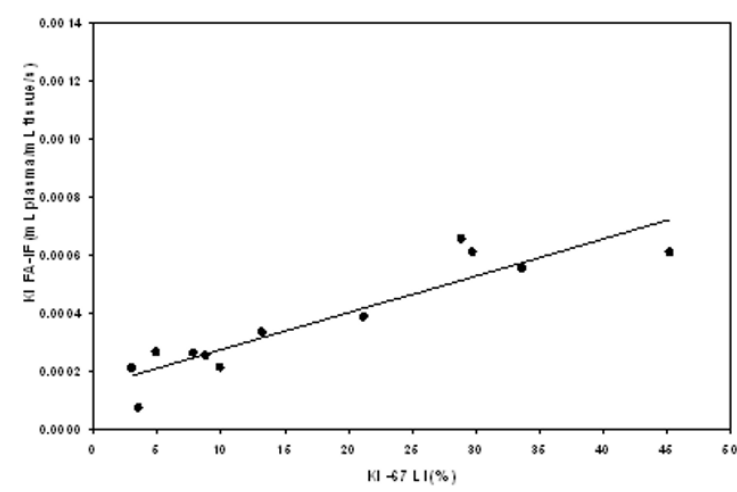

c)

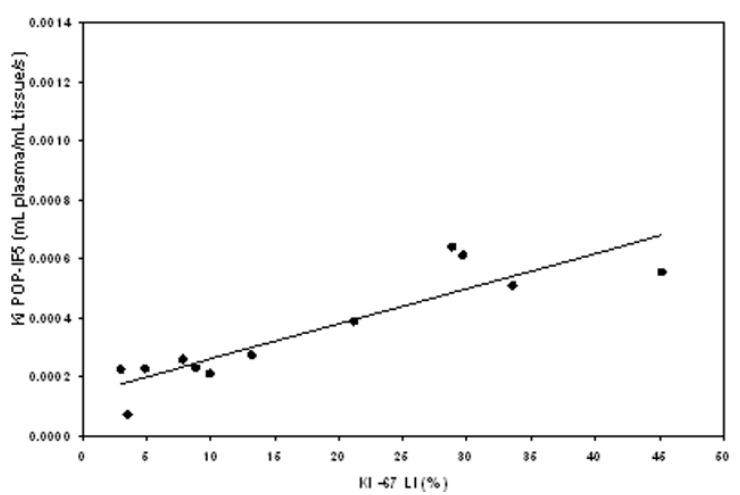

b)

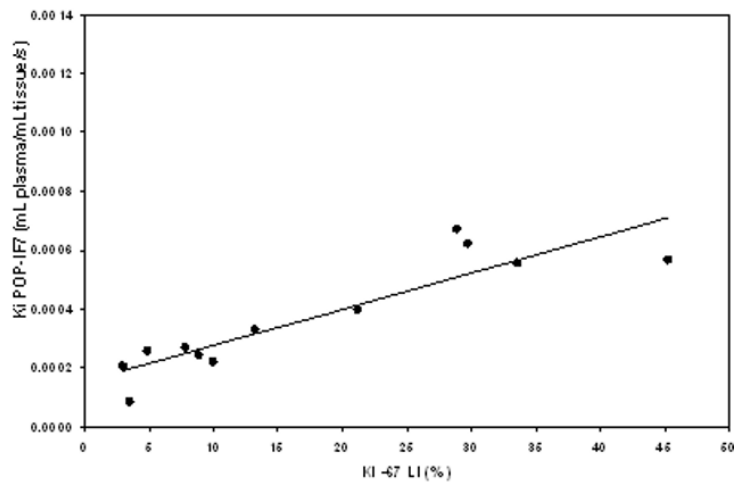

d)

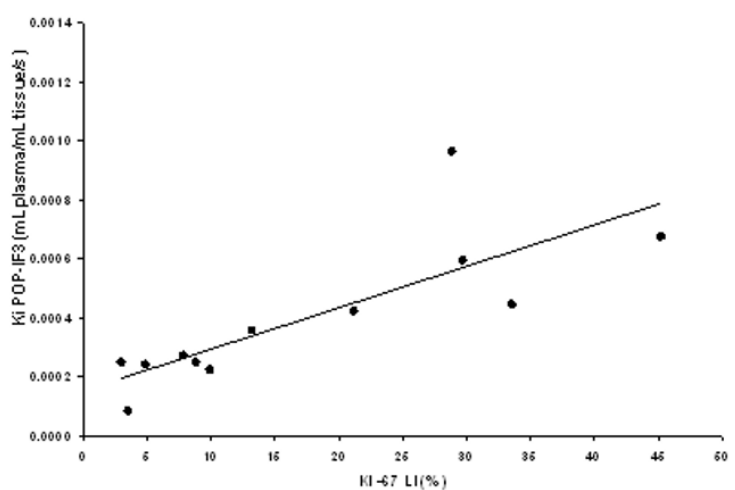

Figure 4 The correlation between Ki-67 LI and modified Patlak Ki. Correlation between Ki-67 LI and modified Patlak Ki using (a) FA-IF ( $\mathrm{R}^{2}=$ 85), (b) POP-IF7 $\left(R^{2}=81\right)$, (c) POP-IF5 $\left(R^{2}=80\right)$ and (d) POP-IF3 $\left(R^{2}=64\right)$.

FLT image-derived input functions reported similar results in reproducibility tests, correlation with $\mathrm{Ki}-67$ and comparison with FA-IFs [21-23]; however, not all tumours will be located in regions with a prominent artery or heart, careful post processing of the raw data may be needed for partial volume and spill over correction given the size of the vessels, which additionally renders these input functions more sensitive to noise and movement. Blood sampling may, in any case, be necessary for metabolite correction of plasma data in compartmental based models.

Venous samples or arterialised venous samples will be easier for patients to tolerate, and compliance is likely to be higher. Considerable effort has been directed at investigating FLT pharmacokinetics in venous and arterial blood. It has been shown that the concentration of FLT in venous plasma samples is systematically marginally higher than the concentration in arterial plasma samples, but this was not found to make a statistically significant difference in analyses $[20,21]$. Shields et al. showed that [14] venous input functions exhibited good correlation with the aortic image-based IF, and venous samples have been used to correct aortic image-based IF for plasma over blood ratio and plasma metabolite fraction [22]. These studies justify the use of venous blood measurements of FLT-PET for the purpose of $K_{i}$ quantification. For these reasons, we expect that population input functions derived using plasma and metabolite correction from venous blood samples, a much more convenient method in clinical settings as opposed to arterial sampling, may yield similar accuracy of Ki values and could make FLT kinetic estimation in tumours even more widely applicable.

\section{Conclusions}

Population-based input functions scaled using limited sampling protocols (consisting of seven, five or three discrete blood measurements), yielded results comparable to the ones obtained using continuous arterial sampling. Consequently $K_{i}$, the physiologically relevant rate 
constant that quantifies tumour radiotracer retention in FLT-PET scans, may be estimated with a small loss of accuracy using a short $(60 \mathrm{~min})$ scan protocol without continuous arterial sampling.

The appropriateness of a population input function and highly reduced blood protocol approach will depend on the purpose of the FLT-PET study in question, as the practical advantages of reducing the number of sampling points is offset by an increasing loss of accuracy in the Ki estimation. The lack of metabolite correction reduced the accuracy of the Ki estimation further; however, the correlation with $\mathrm{Ki}-67$ was still comparable with the one obtained with SUV values.

\section{Appendix}

Schematic derivation of the POP-IFs used in this study.

$$
\begin{aligned}
& \operatorname{POP} \_I F 7(t)=\operatorname{Cp}(t) \cdot\left(1-\frac{x_{1}+x_{2} \cdot t_{\text {parent }}}{\left(x_{3} / t_{\text {parent }}\right)^{x_{4}}+1}\right) \\
& \text { where } \\
& \operatorname{Cp}(t)=\operatorname{ID} \cdot \operatorname{AVGB}(t) \cdot\left(x_{1} \cdot \exp \left(-x_{2} t_{\mathrm{pob}}\right)+x_{3}\right) \quad 0 \leq t \leq \tau \\
& \mathrm{Cp}(t)=\text { spline }\left(t_{\text {plasma }}\right) \quad t>\tau \\
& \operatorname{POP} \_ \text {IF5 }(t)=\operatorname{Cp}(t) \cdot\left(1-\frac{x_{1}+x_{2} \cdot t_{\text {parent }}}{\left(x_{3} / t_{\text {parent }}\right)^{x_{4}}+1}\right) \\
& \text { where } \\
& \mathrm{Cp}(t)=\operatorname{ID} \cdot \operatorname{AVGB}(t) \cdot\left(x_{1} \cdot \exp \left(-x_{2} t_{p o b}\right)+x_{3}\right) \quad 0 \leq t \leq \tau \\
& \mathrm{Cp}(t)=\operatorname{spline}\left(t_{\text {plasma }}\right)+x_{4} \quad t>\tau \\
& \text { POP_IF3 }(t)=\mathrm{Cp}(t) \cdot\left(x_{1} \cdot \exp \left(-x_{2} t_{\text {parent }}\right)+x_{3}\right) \\
& \text { where } \\
& \mathrm{Cp}(t)=\operatorname{ID} \cdot \operatorname{AVGB}(t) \cdot\left(x_{1} \cdot \exp \left(-x_{2} t_{\mathrm{pob}}\right)+x_{3}\right) \quad 0 \leq t \leq \tau \\
& \mathrm{Cp}(t)=\left(x_{1} \cdot \exp \left(-x_{2} t_{\text {plasma }}\right)+x_{3} \cdot \exp \left(-x_{4} t_{\text {plasma }}\right)+x_{5}\right) \quad t>\tau \\
& \text { POP_IF3M }(t)=\mathrm{Cp}(t) \\
& \text { where } \\
& \mathrm{Cp}(t)=\mathrm{ID} \cdot \operatorname{AVGB}(t) \cdot\left(x_{1} \cdot \exp \left(-x_{2} t_{\mathrm{pob}}\right)+x_{3}\right) \quad 0 \leq t \leq \tau \\
& \mathrm{Cp}(t)=\left(x_{1} \cdot \exp \left(-x_{2} t_{\text {plasma }}\right)+x_{3} \cdot \exp \left(-x_{4} t_{\text {plasma }}\right)+x_{5}\right) \quad t>\tau
\end{aligned}
$$

where $\operatorname{AVGB}(t)$ is the average dose-normalised blood function calculated over all dose normalised pre-treatment blood input functions excluding the blood data of the patient to be analysed, ID is the injected dose, $x_{i}$ are the generic parameters of function interpolation, $t_{\mathrm{pob}}$ are the measurement of plasma over blood radioactivity concentration ratio, $t_{\text {plasma }}$ are the measurements of plasma radioactivity concentration, $t_{\text {parent }}$ are the measurements of the plasma fraction of unmetabolised FLT and $\tau$ is a value close to150s.

\section{Acknowledgements}

This study was supported by the United Kingdom Medical Research Council grant (U1200.02.005.00001.01), Cancer Research UK grant (C37/A5610; C2536/ A10337), Experimental Cancer Medicines Centres grant (C37/A7283) and Biomedical Research Centre (BRC) award to the Imperial College Academic Health Sciences Centre (AHSC). The authors would also like to thank the radiographers and chemists at Hammersmith IMANET ${ }^{\circledR}$ for help in production of FLT and PET scanning.

\section{Author details}

${ }^{1}$ Department of Surgery and Cancer, Imperial College Healthcare NHS Trust, Hammersmith Hospital, London, UK ${ }^{2}$ Department of Neuroscience, Imperial College London, London, UK ${ }^{3}$ Imperial College School of Medicine, Room 240, MRC Cyclotron Building, Clinical Sciences Centre, Hammersmith Hospital, Du Cane Road, London W12 0NN, UK

\section{Authors' contributions}

$L R$ and EOA designed the study. KBC, LMK and CRC carried out the clinical aspects of the study. LR, KBC and FET carried out the analytical aspects of the study including statistical analysis and modelling. LR, KBC, EOA drafted the manuscript. All authors read and approved the final manuscript.

\section{Competing interests}

The authors declare that they have no competing interests.

Received: 21 November 2011 Accepted: 24 March 2012

Published: 24 March 2012

\section{References}

1. Shields AF, Grierson JR, Dohmen BM, Machulla HJ, Stayanoff JC, LawhornCrews JM, et al: Imaging proliferation in vivo with [F-18]FLT and positron emission tomography. Nat Med 1998, 4(11):1334-1336.

2. Rudin M: Molecular Imaging-Basic Principles and Applications in Biomedical Research London: Imperial College Press; 2005.

3. Buck AK, Halter G, Schirrmeister H, Kotzerke J, Wurziger I, Glatting G, et al: Imaging proliferation in lung tumors with PET: 18F-FLT versus 18F-FDG. J Nucl Med 2003, 44(9):1426-1431.

4. Ullrich R, Backes H, Li H, Kracht L, Miletic H, Kesper K, et al: Glioma proliferation as assessed by 3'-fluoro-3'-deoxy-L-thymidine positron emission tomography in patients with newly diagnosed high-grade glioma. Clin Cancer Res 2008, 14:2049-2055.

5. Kenny LM, Vigushin DM, Al-Nahhas A, Osman S, Luthra SK, Shousha S, et al: Quantification of cellular proliferation in tumor and normal tissues of patients with breast cancer by [F-18]fluorothymidine-positron emission tomography imaging: evaluation of analytical methods. Cancer Res 2005, 65(21):10104-10112.

6. Buck AK, Bommer M, Stilgenbauer S, Juweid M, Glatting G, Schirrmeister $H_{\text {, }}$ et al: Molecular imaging of proliferation in malignant lymphoma. Cancer Res 2006, 66(22):11055-11061.

7. Mourik JEM, van Velden FHP, Lubberink M, Kloet RW, van Berckel BNM, Lammertsma AA, et al: Image derived input functions for dynamic high resolution research tomograph PET brain studies. Neuroimage 2008, 43(4):676-686.

8. Germano G, Chen BC, Huang SC, Gambhir SS, Hoffman EJ, Phelps ME: Use of the abdominal aorta for arterial input function determination in hepatic and renal PET studies. J NuCl Med 1992, 33(4):613-620.

9. Watabe H, Channing MA, Riddell C, Jousse F, Libutti SK, Carrasquillo JA, et al: Noninvasive estimation of the aorta input function for measurement of tumor blood flow with. IEEE Trans Med Imaging 2001, 20(3):164-174.

10. Asselin MC, Cunningham VJ, Amano S, Gunn RN, Nahmias C: Parametrically defined cerebral blood vessels as non-invasive blood input functions for brain PET studies. Phys Med Biol 2004, 49(6):1033-1054.

11. Zanotti-Fregonara P, Fadaili EM, Maroy R, Comtat C, Souloumiac A, Jan S, et al: Comparison of eight methods for the estimation of the imagederived input function in dynamic [(18)F]-FDG PET human brain studies. J Cereb Blood Flow Metab 2009, 29(11):1825-1835.

12. Sundaram SK, Freedman NMT, Carrasquillo JA, Carson JM, Whatley M, Libutti SK, et al: Simplified kinetic analysis of tumor 18F-FDG uptake: a dynamic approach. J Nucl Med 2004, 45(8):1328-1333.

13. Bentourkia M: Kinetic modeling of PET data without blood sampling. IEEE 2005, 52(3):697-702.

14. Shields AF, Briston DA, Chandupatla S, Douglas KA, Lawhorn-Crews J, Collins JM, et al: A simplified analysis of $\left[{ }^{18} \mathrm{~F}\right] 3^{\prime}$-deoxy-3'-fluorothymidine metabolism and retention. Eur J NuCl Med Mol Imaging 2005, 32(11):1269-1275. 
15. Cook GJ, Lodge MA, Marsden PK, Dynes A, Fogelman I: Non-invasive assessment of skeletal kinetics using fluorine-18 fluoride positron emission tomography: evaluation of image and population-derived arterial input functions. Eur J Nucl Med 1999, 26(11):1424-1429.

16. Takikawa S, Dhawan V, Spetsieris P, Robeson W, Chaly T, Dahl R, et al: Noninvasive quantitative fluorodeoxyglucose PET studies with an estimated input function derived from a population-based arterial blood curve. Radiology 1993, 188(1):131-136.

17. Eberl S, Anayat AR, Fulton RR, Hooper PK, Fulham MJ: Evaluation of two population-based input functions for quantitative neurological FDG PET studies. Eur J Nucl Med 1997, 24(3):299-304.

18. Kissel J, Port RE, Zaers J, Bellemann ME, Strauss LG, Haberkorn U, et al: Noninvasive determination of the arterial input function of an anticancer drug from dynamic PET scans using the population approach. Med Phys 1999, 26(4):609-615.

19. Kim SJ, Lee JS, Im KC, Kim SY, Park SA, Lee SJ, et al: Kinetic modeling of 3 '-deoxy-3 '-F-18-fluorothymidine for quantitative cell proliferation imaging in subcutaneous tumor models in mice. J Nucl Med 2008 49(12):2057-2066.

20. Menda Y, Ponto LLB, Dornfeld KJ, Tewson TJ, Watkins GL, Schultz MK, et a Kinetic analysis of 3 '-deoxy-3 '-F-18-fluorothymidine (F-18-FLT) in head and neck cancer patients before and early after initiation of chemoradiation therapy. J Nucl Med 2009, 50(7):1028-1035.

21. Visvikis D, Francis D, Mulligan R, Costa DC, Croasdale I, Luthra SK, et al: Comparison of methodologies for the in vivo assessment of (FLT)-F-18 utilisation in colorectal cancer. Eur J Nucl Med Mol Imaging 2004, 31(2):169-178

22. de Langen AJ, Klabbers B, Lubberink M, Boellaard R, Spreeuwenberg MD, Slotman BJ, et al: Reproducibility of quantitative F-18-3'-deoxy-3'fluorothymidine measurements using positron emission tomography. Eur J Nucl Med Mol Imaging 2009, 36(3):389-395.

23. Backes $H$, Ullrich R, Neumaier B, Kracht L, Wienhard K, Jacobs AH: Noninvasive quantification of F-18-FLT human brain PET for the assessment of tumour proliferation in patients with high-grade glioma. Eur J Nucl Med Mol Imaging 2009, 36(12):1960-1967.

24. Kenny L, Coombes RC, Vigushin DM, Al-Nahhas A, Shousha S, Aboagye EO Imaging early changes in proliferation at 1 week post chemotherapy: a pilot study in breast cancer patients with $3^{\prime}$-deoxy $-3^{\prime}-\left[{ }^{18} \mathrm{~F}\right]$ fluorothymidine positron emission tomography. Eur J Nucl Med Mol Imaging 2007, 34(9):1339-1347.

25. Coleman TF, Li Y: On the convergence of reflective Newton methods for large-scale nonlinear minimization subject to bounds. 1994, 67(2):189-224

26. Coleman TF, Li Y: An interior, trust region approach for nonlinear minimization subject to bounds. SIAM J Optim 1996, 6:418-445.

27. Muzi M, Mankoff DA, Grierson JR, Wells JM, Vesselle H, Krohn KA: Kinetic modeling of 3 '-deoxy-3'-fluorothymidine in somatic tumors: mathematical studies. J Nucl Med 2005, 46(2):371-380.

28. Mankoff DA, Shields AF, Graham MM, Link JM, Krohn KA: A graphical analysis method to estimate blood-to-tissue transfer constants for tracers with labeled metabolites. J Nucl Med 1996, 37(12):2049-2057.

doi:10.1186/2191-219X-2-11

Cite this article as: Contractor et al:: Evaluation of limited blood sampling population input approaches for kinetic quantification of $\left[{ }^{18} \mathrm{~F}\right]$ fluorothymidine PET data. EJNMMI Research 2012 2:11.

\section{Submit your manuscript to a SpringerOpen ${ }^{\mathcal{O}}$ journal and benefit from:}

- Convenient online submission

- Rigorous peer review

- Immediate publication on acceptance

- Open access: articles freely available online

- High visibility within the field

- Retaining the copyright to your article

Submit your next manuscript at $\gg$ springeropen.com 